Volume 2

Issue 4 -- Integrative Medicine

Article 28

$11-20-2015$

\title{
Mailed At-Home FIT Intervention to Increase Colorectal Screenings at Sixteenth Street Community Health Centers
}

\author{
Alexander V. Herrera \\ Brian Hilgeman \\ Michelle Buelow \\ Melissa A. Lemke
}

Follow this and additional works at: https://aah.org/jpcrr

Part of the Community Health and Preventive Medicine Commons, Oncology Commons, and the Public Health Education and Promotion Commons

\section{Recommended Citation}

Herrera AV, Hilgeman B, Buelow M, Lemke MA. Mailed At-Home FIT Intervention to Increase Colorectal Screenings at Sixteenth Street Community Health Centers. J Patient Cent Res Rev 2015;2:212.

http://dx.doi.org/10.17294/2330-0698.1238

Published quarterly by Midwest-based health system Advocate Aurora Health and indexed in PubMed Central, the Journal of Patient-Centered Research and Reviews (JPCRR) is an open access, peer-reviewed medical journal focused on disseminating scholarly works devoted to improving patient-centered care practices, health outcomes, and the patient experience. 
nonselectively, inhibits fungal growth in soil samples at moderately high levels. It does not appear likely that this Scedosporium apiospermum strain employs $\beta$-Th resistance for selective advantage in cedar mulched landscaping.

\section{Mailed At-Home FIT Intervention to Increase Colorectal Screenings at Sixteenth Street Community Health Centers}

\author{
Alexander V. Herrera, Brian Hilgeman, Michelle Buelow, \\ Melissa A. Lemke
}

TRIUMPH Program, University of Wisconsin School of Medicine and Public Health; Internal Medicine and Family Medicine, Sixteenth Street Community Health Center

Background: Mailed at-home FIT intervention kits to increase colorectal cancer screenings at Sixteenth Street Community Health Centers (SSCHC).

Purpose: It is our goal to increase the current SSCHC colorectal cancer baseline screening rate of $23 \%$ to $50 \%$ within three years of full at-home FIT kit implementation.

Methods: Colon cancer is the second and third most common cause of cancer death in the United States in Hispanic men and women, respectively. Colonoscopy is the most common method of colon cancer screening, even among low-income patients. However, it has been shown in community health centers that mailed FIT kits are a more effective outreach method (40.7\% completion) than colonoscopy outreach $(24.6 \%)$ or usual care $(12.1 \%)$. We hope to increase colorectal cancer screening in eligible patients at the SSCHC through mailed at-home FIT kits that have FIT materials, instructions and educational materials based on the Health Belief Model. Results: A trial intervention will assess the potential for annual implementation with hopes of full implementation to all of SSCHC eligible patients in the future.

Conclusion: Application of culturally relevant interventions can be a practical and inexpensive method of increasing colorectal screening rates in community health centers with predominantly Hispanic populations.

\section{Maternal Intuition of Fetal Gender}

Michael P. McFadzen, David P. Dielentheis, Ronda Kasten

\section{Department of Obstetrics, Aurora Sheboygan Clinic}

Background: Many pregnant mothers feel they have a perception or intuition as to the gender of their unborn baby. There is very little published scientific literature regarding this topic. The study's goal is to determine accuracy of mothers' perceptions as to gender of their unborn babies. Many scientists believe a pregnant woman could not determine her baby's gender by intuition, with a $50 \%$ probability of correctly determining the gender. This study should be considered fun science.

Purpose: To objectively measure a pregnant mother's perception as to the gender of her unborn baby and compare to sonographically proved gender. The study also will measure the percentage of pregnant patients who have this intuition.

Methods: All patients will be presenting for their secondtrimester screening ultrasound in the Obstetrics Department of Aurora Sheboygan Clinic and must be 17-23 weeks pregnant. A medical sonographer will describe the ultrasound exam and obtain appropriate consent and medical history. The patient will be asked if they have perception as to the fetal gender; their answer will be logged. Patients with knowledge of fetal gender will be excluded from this study. Results: Thus far, 128 patients have qualified for the study (with an expected cohort of 400). Approximately one-third of our patient population has "intuition" or "perception" on the gender of their baby. Of these, $47 \%$ correctly indicated fetal gender, 53\% did not. Within this study, we've started categorizing patients who have a strong intuition of fetal gender. This cohort has correctly indicated gender with $90 \%$ accuracy; however, there are not enough participants for clinical relevancy at this point in the study.

Conclusion: Preliminary data indicates mom perception of fetal gender is $47 \%$ accurate.

\section{Disease-Management in Family Medicine Clinics Through the Addition of a Health Coach: A Pilot Study}

Crystal Y. Cichon, Jessica J.F. Kram, Tiffany A. Mullen, Pamela Voelkers, Kristin J. Magliocco, Kiley A. Bernhard, Dennis J. Baumgardner

Department of Family Medicine, Aurora Health Care; Center for Urban Population Health; Aurora Advanced Health Care; University of Wisconsin School of Medicine and Public Health; Aurora UW Medical Group

Background: In the United States, more than $80 \%$ of health care spending is focused on the management of chronic illnesses such as hypertension, diabetes and hyperlipidemia. Controlling these chronic diseases can lead to better health outcomes and decrease the number of preventable deaths. Patient self-management has shown to improve clinical outcomes. In a primary care setting, a multidisciplinary approach can more effectively educate patients on improving their health.

Purpose: To assess the impact of a health coach in a primary care setting as it relates to clinical outcomes.

Methods: Patients from two Aurora family medicine clinics were referred to a health coach by primary care providers. A total of 40 patients participated and paid out of pocket for the health coaching sessions (intervention). Patients had at least one scheduled session with the health coach that covered topics such as healthy eating, weight loss and exercise. Patient data, including glycohemoglobin, lipid panels and blood pressures, were reviewed pre- and postintervention. 